\title{
Optimal AC Distribution Systems Reconfiguration
}

\author{
Hassan L. Hijazi and Sylvie Thiébaux \\ NICTA \\ Optimisation Research Group \\ The Australian National University \\ Canberra, ACT 2601, Australia \\ Email: hassan.hijazi@nicta.com.au,sylvie.thiebaux@nicta.com.au
}

\begin{abstract}
Convex power flow relaxations have become popular to alleviate difficulties with embedding the non-convex AC power flow equations into optimisation models, and to provide guarantees on the quality of feasible solutions generated by heuristic approaches. However, their use has almost universally been limited to purely continuous problems. This paper extends the reach of relaxations to reconfiguration problems with binary decision variables, such as minimal power loss, load balancing and power supply restoration. This is achieved by extending the relaxations of $\mathrm{AC}$ power flows to bear on the on/off nature of constraints featured in reconfiguration problems. This leads to an approach producing $\mathrm{AC}$ feasible solutions with provable optimality gaps, and to global optimal solutions in some cases. In terms of run time, the new models are competitive with stateof-the-art approximations which lack formal guarantees.
\end{abstract}

Keywords-Distribution Systems Reconfiguration, Power Supply Restoration, AC Power Flow, DC Power Flow, On/Off Constraints, Global Optimisation, Convex Relaxations, MINLP

\section{INTRODUCTION}

The steady-state alternating current $(\mathrm{AC})$ power flow equations are at the core of virtually every computational problem in the field of power systems. Unfortunately, these equations form a system of non-convex constraints, raising significant challenges in any optimisation framework. When embedding these constraints into optimisation models, global non-linear programming (NLP) solvers do not scale and may not converge, even if all decision variables are continuous. These issues are exacerbated in the case of reconfiguration and power supply restoration problems, where discrete decisions are made that affect the grid topology. On the one hand, discrete variables increase the computational difficulty of the problem. On the other hand, topology changes lead to departure from the normal operating conditions that are used to hot-start non-linear solvers. Yet utilities are facing an increasing need for efficient reconfiguration methods, in order to optimise their operations to better handle the intermittent nature of distributed generation, and to quickly resupply customers in fault situations.

There is a rich set of optimisation approaches in the power systems literature that tries to circumvent these problems. Black-box heuristic methods, which push power flow calculations outside the optimisation solver, have become very popular, owing to their broad applicability [1]-[3]. Yet, these approaches fail to exploit the problem structure and lack formal guarantees on the quality of the solutions returned. Another popular approach is to approximate the power flow equations, usually linearly [4]-[6], or with more accurate convex models [7]. This enables the use of a new generation of mathematical programming solvers including mixed-integer linear (MIP), quadratic (QP) and second-order cone (SOCP) programming solvers [8]. With these approaches, the issue is that the solution returned - whilst optimal for the approximate model - may not be feasible with respect to the original AC model. Researchers have observed that this is particularly true with reconfiguration problems, as switching moves us away from the nominal configuration under which the approximations are valid [9].

Therefore, recent research has turned to finding linear or convex relaxations of the AC power flow equations [10][13]. These convex relaxations are of great interest since they efficiently produce lower bounds on the quality of the feasible solutions generated by global or local optimisation approaches. For purely continuous problems such as optimal power flow (OPF), the semi-definite programming (SDP) relaxation in [12] is often tight, i.e. its solution is also a solution to the original problem. However, for reconfiguration and power supply restoration problems, the applicability of such relaxations has not been investigated; the only exception we are aware of is the work of Jabr et. al. [14] discussed below. Such an investigation is the topic of the present paper.

Our contributions are as follows. We extend recent quadratic relaxations of power flow equations for radial [11] and general networks [13] to include topology changes and bear on reconfiguration problems. Key to obtaining effective relaxations is an adequate representation of on/off constraints, which we describe in some detail. Studying both radial and non-radial topologies enables us to efficiently exploit the current radial topology of most distribution systems, whilst also accounting for more general topologies representative of advanced networks and microgrids with higher penetration of distributed generation. We then use these relaxations to obtain lower bounds and solve reconfiguration problems with objectives such as loss minimisation and load balancing, as in [7], as well as power supply restoration problems.

Qualitatively, our approach leads to AC feasible solutions with provable optimality gaps, of zero in some cases. Except for the work of Jabr et. al [14], we are not aware of other approaches capable of providing such guarantees for reconfiguration problems. Jabr et. al use a different relaxation to solve loss minimisation problems, exclusively with radial topologies. In the meshed case, and for power supply restoration (regardless of topology), we are not aware of any report of provable quality gaps. Quantitatively, for radial topologies, our approach leads to better computation times when compared to existing approaches based on Tabu search [15], SOCP with convex power flow approximations [7], and MICP using the competing relaxation [14]. Our experimental results also suggest that meshed topologies are often beneficial: in the benchmarks we 
considered, we observed a further reduction of the objective function between $2 \%-15 \%$.

The paper is organised as follows. Section II reviews the power flow equations suited to the radial and general case, as well as their relaxation given in [11] and [13], respectively. Section III extends these relaxations to account for the specificities of reconfiguration problems and describes our overall approach. Section IV describes the three specific reconfiguration problems we consider, and presents an experimental evaluation of our approach on these problems.

\section{POWER Flow Equations ANd Their Relaxation}

In the following, we consider a network $\langle N, E\rangle$, where $N$ is the set of buses (nodes), and $E$ is the set of lines (edges). The relevant variables are the voltage magnitude $v_{i}$, the phase angle $\theta_{i}$ at each bus $i \in N$, the active power $p_{i j}$ and the reactive power $q_{i j}$ from bus $i$ to bus $j$. Each line has a constant impedance, which we represent either via its resistance $\boldsymbol{r}_{i j}$ and reactance $\boldsymbol{x}_{i j}$ or via its susceptance $\boldsymbol{b}_{i j}$ and conductance $\boldsymbol{g}_{i j}$.

We assume the following set of operational constraints, where $\boldsymbol{S}_{i j}^{u}$ is the capacity of line $(i, j) \in E, \boldsymbol{\theta}^{u}$ is the maximal phase angle difference between connected buses, and $\left(\boldsymbol{v}^{l}, \boldsymbol{v}^{u}\right)$ are the lower and upper bus voltage limits

$$
\begin{aligned}
& p_{i j}^{2}+q_{i j}^{2} \leqslant \boldsymbol{S}_{i j}^{u},(i, j) \in E \\
& -\boldsymbol{\theta}^{u} \leqslant \theta_{i}-\theta_{j} \leqslant \boldsymbol{\theta}^{u},(i, j) \in E \\
& \boldsymbol{v}^{l} \leqslant v_{i} \leqslant \boldsymbol{v}^{u}, i \in N .
\end{aligned}
$$

\section{A. Radial Distribution Networks}

Distribution systems have a meshed structure, but are often exploited radially. From an optimisation point of view, the radial topology makes it possible to exploit the quadratic DistFlow equations [1], resulting in more efficient computation. These equations are defined as

$$
\begin{aligned}
p_{i j} & =\sum_{k:(j, k) \in E} p_{j k}+\boldsymbol{r}_{i j} \frac{p_{i j}^{2}+q_{i j}^{2}}{v_{i}^{2}} \\
q_{i j} & =\sum_{k:(j, k) \in E} q_{j k}+\boldsymbol{x}_{i j} \frac{p_{i j}^{2}+q_{i j}^{2}}{v_{i}^{2}} \\
v_{j}^{2} & =v_{i}^{2}-2\left(\boldsymbol{r}_{i j} p_{i j}+\boldsymbol{x}_{i j} q_{i j}\right)+\left(\boldsymbol{r}_{i j}^{2}+\boldsymbol{x}_{i j}^{2}\right) \frac{p_{i j}^{2}+q_{i j}^{2}}{v_{i}^{2}}
\end{aligned}
$$

We use the relaxation of the DistFlow equations described by Farivar et. al [11]. The relaxation substitutes $v_{i}^{2}$ with the variable $v v_{i}$ which denotes the square of the voltage magnitude at each bus, and defines the variables

$$
l_{i j}=\frac{p_{i j}^{2}+q_{i j}^{2}}{v v_{i}}
$$

representing the square of the current magnitude on each line. This makes all equations linear except for the latter equality which is the source of non-convexity and is relaxed into an inequality. The overall relaxation is

$$
\begin{aligned}
& p_{i j}=\sum_{k:(j, k) \in E} p_{j k}+\boldsymbol{r}_{i j} l_{i j} \\
& q_{i j}=\sum_{k:(j, k) \in E} q_{j k}+\boldsymbol{x}_{i j} l_{i j} \\
& v v_{j}=v v_{i}-2\left(\boldsymbol{r}_{i j} p_{i j}+\boldsymbol{x}_{i j} q_{i j}\right)+\left(\boldsymbol{r}_{i j}^{2}+\boldsymbol{x}_{i j}^{2}\right) l_{i j} \\
& p_{i j}^{2}+q_{i j}^{2} \leqslant l_{i j} v v_{i}
\end{aligned}
$$

This relaxation was used by Farivar et. al [11] for Volt/VAR control; we will extend it to the case of reconfiguration. In solving a range of reconfiguration problems including loss minimisation and load balancing, Taylor and Hover [7] have used a SOCP approximation of the DistFlow equations which ignores the last term in Equation (2c). This approximation does not come with guarantees on the feasibility and quality of the solution returned. The alternative relaxation proposed by Jabr et. al [14], will also be a point of comparison in our experimental section.

\section{B. General Distribution Networks}

As the penetration of distributed generation increases, distribution networks will progressively evolve into active meshed networks with bi-directional power flows. Therefore, this paper also considers networks with general meshed topologies. The steady-state $\mathrm{AC}$ power flows in such networks are given by

$$
\begin{gathered}
p_{i j}=\boldsymbol{g}_{i j} v_{i}^{2}-\boldsymbol{g}_{i j} v_{i} v_{j} \cos \left(\theta_{i}-\theta_{j}\right)-\boldsymbol{b}_{i j} v_{i} v_{j} \sin \left(\theta_{i}-\theta_{j}\right) \\
q_{i j}=-\boldsymbol{b}_{i j} v_{i}^{2}+\boldsymbol{b}_{i j} v_{i} v_{j} \cos \left(\theta_{i}-\theta_{j}\right)-\boldsymbol{g}_{i j} v_{i} v_{j} \sin \left(\theta_{i}-\theta_{j}\right)
\end{gathered}
$$

Finding an effective relaxation of these equations is much more challenging than in the radial case. We use the recent convex quadratic relaxation of the $\mathrm{AC}$ power flow equations proposed by Hijazi et. al [13], which offers a number of advantages over other relaxations [12], [16]. In particular, SDP solvers required by the SDP relaxation [12] are less mature than non-linear quadratic solvers in terms of scalability. Furthermore, preliminary experiments have proven [13] to be a tighter relaxation than [16].

Here we only mention the main principles behind the relaxation and refer the reader to [13] for further details. As shown in Figure 1, the formulation exploits convex relaxations of the non-linear terms appearing in equations (4a)-(4b). It uses:

- a quadratic relaxation of cosine (for $\boldsymbol{\theta}^{u} \leqslant \frac{\pi}{2}$ )

$$
\langle\cos (\theta)\rangle^{R}=\left\{\begin{array}{l}
\widetilde{c_{i j}} \leqslant 1-\frac{1-\cos \left(\boldsymbol{\theta}^{u}\right)}{\left(\boldsymbol{\theta}^{u}\right)^{2}} \theta^{2} \\
\widetilde{c_{i j}} \geqslant \cos \left(\boldsymbol{\theta}^{u}\right)
\end{array}\right.
$$

- $\quad$ a quadratic relaxation of $v^{2}$

$$
\left\langle v^{2}\right\rangle^{R}=\left\{\begin{array}{l}
\widetilde{v v} \geqslant v^{2} \\
\widetilde{v v} \leqslant\left(\boldsymbol{v}^{u}+\boldsymbol{v}^{l}\right) v-\boldsymbol{v}^{u} \boldsymbol{v}^{l}
\end{array}\right.
$$

- a polyhedral relaxation of sine (for $\boldsymbol{\theta}^{u} \leqslant \frac{\pi}{2}$ )

$$
\langle\sin (\theta)\rangle^{R}=\left\{\begin{array}{l}
\widetilde{s_{i j}} \leqslant \cos \left(\frac{\boldsymbol{\theta}^{u}}{2}\right)\left(\theta-\frac{\boldsymbol{\theta}^{u}}{2}\right)+\sin \left(\frac{\boldsymbol{\theta}^{u}}{2}\right) \\
\widetilde{s_{i j}} \geqslant \cos \left(\frac{\boldsymbol{\theta}^{u}}{2}\right)\left(\theta+\frac{\boldsymbol{\theta}^{u}}{2}\right)-\sin \left(\frac{\boldsymbol{\theta}^{u}}{2}\right)
\end{array}\right.
$$

- $\quad$ and the McCormick relaxation of bilinear terms $v w$

$$
\langle v w\rangle^{M}=\left\{\begin{array}{l}
\widetilde{v w} \geqslant \boldsymbol{v}^{l} w+\boldsymbol{w}^{l} v-\boldsymbol{v}^{l} \boldsymbol{w}^{l} \\
\overline{v w} \geqslant \boldsymbol{v}^{u} w+\boldsymbol{w}^{u} v-\boldsymbol{v}^{u} \boldsymbol{w}^{u} \\
\widetilde{v w} \leqslant \boldsymbol{v}^{l} w+\boldsymbol{w}^{u} v-\boldsymbol{v}^{l} \boldsymbol{w}^{u} \\
\widetilde{v w} \leqslant \boldsymbol{v}^{u} w+\boldsymbol{w}^{l} v-\boldsymbol{v}^{u} \boldsymbol{w}^{l}
\end{array}\right.
$$

The relaxation is strengthened by introducing the following formulation of power loss equations [17]

$$
\begin{aligned}
p_{i j}+p_{j i} & =\boldsymbol{r}_{i j} l_{i j} \\
q_{i j}+q_{j i} & =\boldsymbol{x}_{i j} l_{i j}
\end{aligned}
$$



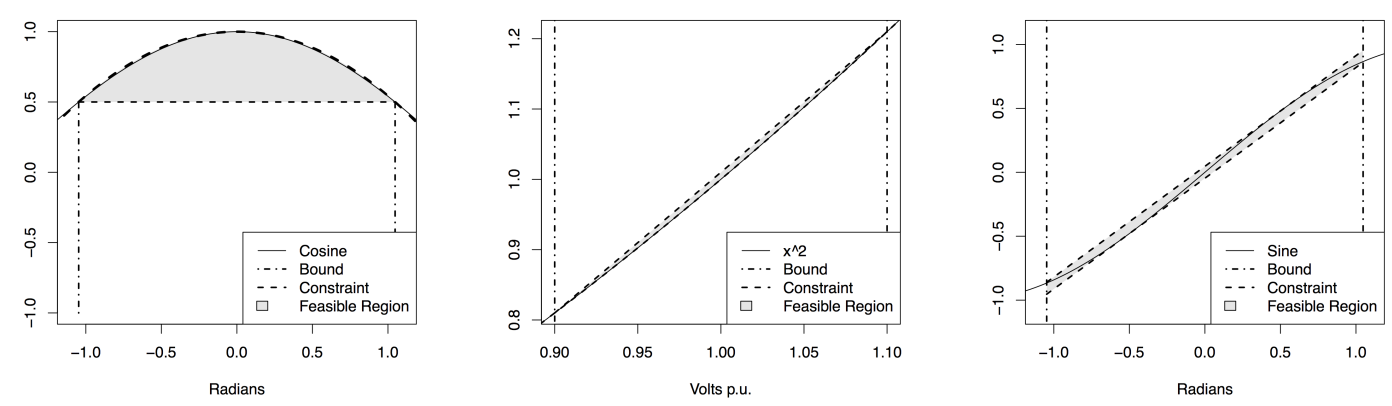

Fig. 1. Relaxation of Non-linear Functions (from [13]): cosine (left), square (middle), and sine (right).

where, as in the radial case, $l_{i j}$ denotes the square of the current magnitude. This builds on the observation that introducing redundancy can often improve relaxations of nonconvex problems [18].

Altogether, the relaxation obtained is

$$
\begin{aligned}
& p_{i j}=\boldsymbol{g}_{i j} \widetilde{v v}_{i}-\boldsymbol{g}_{i j} \widetilde{w c}_{i j}-\boldsymbol{b}_{i j} \widetilde{w s}_{i j} \\
& q_{i j}=-\boldsymbol{b}_{i j} \widetilde{v v}_{i}+\boldsymbol{b}_{i j} \widetilde{w c}_{i j}-\boldsymbol{g}_{i j} \widetilde{w s}_{i j} \\
& \widetilde{v v}_{i} \in\left\langle v^{2}\right\rangle^{R} \\
& \check{c}_{i j} \in\left\langle\cos \left(\theta_{i}-\theta_{j}\right)\right\rangle^{R} \\
& \check{s}_{i j} \in\left\langle\sin \left(\theta_{i}-\theta_{j}\right)\right\rangle^{R} \\
& \breve{w}_{i j} \in\left\langle\breve{v}_{i}, \breve{v}_{j}\right\rangle^{M} \\
& \widetilde{w c}_{i j} \in\left\langle\breve{w}_{i j}, \check{c}_{i j}\right\rangle^{M} \\
& \widetilde{w s}_{i j} \in\left\langle\breve{w}_{i j}, \breve{s}_{i j}\right\rangle^{M} \\
& p_{i j}^{2}+q_{i j}^{2} \leqslant l_{i j} \widetilde{v v}_{i} \\
& p_{i j}+p_{j i}=\boldsymbol{r}_{i j} l_{i j} \\
& q_{i j}+q_{j i}=\boldsymbol{x}_{i j} l_{i j}
\end{aligned}
$$

In [13], this relaxation is used to solve OPF and capacitor placement problems. In the following we extend the relaxation to reconfiguration and demonstrate its benefits for a range of reconfiguration problems.

\section{RELAXATIONS FOR RECONFIGURATION PROBLEMS}

Distribution system reconfiguration involves determining the network topology (the position open/closed of line switches) optimising a given performance criterion whilst satisfying physical and operational constraints. For simplicity, we assume that each line $(i, j) \in E$ is equipped with a switch whose position is given by the (non-directional) boolean variable $y_{i j}$, where $y_{i j}=1$ means that the switch is closed. A line without switch can easily be accommodated by fixing $y_{i j}=1$. In a distribution system, a subset $G \subseteq N$ of buses are substation buses. Each bus $i \in N \backslash G$ has constant active and reactive loads $\boldsymbol{p}_{i}^{l}$ and $\boldsymbol{q}_{i}^{l}$.

For reconfiguration, the set of operational constraints is now the following:

$$
\begin{array}{lr}
p_{i j}^{2}+q_{i j}^{2} \leqslant \boldsymbol{S}_{i j}^{u}, & \text { if } y_{i, j}=1,(i, j) \in E \\
-\boldsymbol{\theta}^{u} \leqslant \theta_{i}-\theta_{j} \leqslant \boldsymbol{\theta}^{u} & \text { if } y_{i, j}=1,(i, j) \in E \\
v_{i}=1, & i \in G \\
\boldsymbol{v}^{l} \leqslant v_{i} \leqslant \boldsymbol{v}^{u}, & i \in N \backslash G .
\end{array}
$$

Observe that with the addition of topology changes, some of the constraints become on/off: they are only active if $y_{i j}$ is true.
For instance, this affects the phase angle constraint (6b), and will also affect some of the equations in the power flow relaxations. The key to efficient formulations and convex relaxations of reconfiguration problems is an adequate representation of these on/off constraints. Therefore, before extending the relaxations, we review on/off constraints representations.

\section{A. On/Off Constraints}

Given convex functions $f: \mathbb{R}^{n+m} \rightarrow \mathbb{R}, \mathbf{h}: \mathbb{R}^{n+m} \rightarrow \mathbb{R}^{q}$ and $g_{k}: \mathbb{R}^{n} \rightarrow \mathbb{R}, \forall k \in K$, we are interested in optimisation problems of the form

$$
\begin{aligned}
\min & f(\mathbf{x}, \mathbf{z}) \\
\text { s.t. } & \mathbf{h}(\mathbf{x}, \mathbf{z}) \leqslant \mathbf{0}, \\
& g_{k}(\mathbf{x}) \leqslant 0 \text { if } z_{k}=1, \forall k \in K \\
& \mathbf{x} \in \mathbb{R}^{n}, \mathbf{z} \in \mathbb{Z}^{m} .
\end{aligned}
$$

Each $g_{k}(\mathbf{x}) \leqslant 0$ represents an "on/off" constraint, with $z_{k}$ as its corresponding indicator variable. $\mathbf{h}(\mathbf{x}, \mathbf{z}) \leqslant \mathbf{0}$ gathers the remaining constraints. Bounds on variables are assumed to be finite. A standard formulation of (Pr) would consist of transforming the "on/off" constraints into: $z_{k} g_{k}(\mathbf{x}) \leqslant 0, \forall k \in$ $K$, loosing the convexity of the feasible region. In order to maintain the convexity property, one can use big-M formulations. Unfortunately the continuous relaxations resulting from such models, although compact and usually easy to solve, often provide weak lower bounds. Disjunctive programming (see [19]) offers an interesting alternative. Indeed, (Pr) can be reformulated as a disjunctive program

$$
\begin{aligned}
\min & f(\mathbf{x}, \mathbf{z}) \\
\text { s.t. } & \mathbf{h}(\mathbf{x}, \mathbf{z}) \leqslant \mathbf{0}, \\
& \left(\mathbf{x}, z_{k}\right) \in \Gamma_{0}^{k} \cup \Gamma_{1}^{k}, \forall k \in K \\
& \Gamma_{0}^{k}=\left\{\left(\mathbf{x}, z_{k}\right): z_{k}=0, \mathbf{l}^{0} \leqslant \mathbf{x} \leqslant \mathbf{u}^{0}\right\} \\
& \Gamma_{1}^{k}=\left\{\left(\mathbf{x}, z_{k}\right): z_{k}=1, g_{k}(\mathbf{x}) \leqslant 0, \mathbf{l}^{1} \leqslant \mathbf{x} \leqslant \mathbf{u}^{1}\right\} . \\
& \mathbf{x} \in \mathbb{R}^{n}, \mathbf{z} \in \mathbb{Z}^{m} .
\end{aligned}
$$

Given this approach, one can define the best convex relaxation of each disjunctive constraint $g_{k}$ to be the convex hull $\operatorname{conv}\left(\Gamma_{0}^{k} \cup \Gamma_{1}^{k}\right)$. When the set $\Gamma_{0}^{k}$ reduces to a single point $\left(\mathbf{l}^{0}=\mathbf{u}^{0}=0\right)$, conv $\left(\Gamma_{0}^{k} \cup \Gamma_{1}^{k}\right)$ can be formulated in the space of original variables [20]. The main result is the following (subscript $k$ is dropped for clarity purposes)

$$
\operatorname{conv}\left(\Gamma_{0} \cup \Gamma_{1}\right)=\operatorname{closure}\left(\Gamma_{c}\right) \text {, where }
$$




$$
\Gamma_{c}=\left\{\begin{array}{l}
(\mathbf{x}, z) \in \mathbb{R}^{n+1}: \\
z g(\mathbf{x} / z) \leqslant 0, \\
z \mathbf{l}^{1} \leqslant \mathbf{x} \leqslant z \mathbf{u}^{1}, 0<z \leqslant 1
\end{array}\right\}
$$

Let us emphasise that for quadratic functions

$$
g(\mathbf{x})=\alpha_{1} x_{1}^{2}+\alpha_{2} x_{2}^{2}-\beta x_{3}
$$

e.g. $p_{i j}^{2}+q_{i j}^{2}-l_{i j}, \Gamma_{c}$ is closed, and the convex hull is

$$
\Gamma_{c}=\left\{\begin{array}{l}
(\mathbf{x}, z) \in \mathbb{R}^{4}: \\
\alpha_{1} x_{1}^{2}+\alpha_{2} x_{2}^{2} \leqslant \beta z x_{3}, \\
z \mathbf{l}^{1} \leqslant \mathbf{x} \leqslant z \mathbf{u}^{1}, 0 \leqslant z \leqslant 1
\end{array}\right\}
$$

This result can be extended to the general case $\left(\mathbf{l}^{0} \neq \mathbf{u}^{0}\right)$ when functions $g$ are monotonic [21]. Specifically, in the linear case where $g(\mathbf{x})=\boldsymbol{\alpha}^{T} \mathbf{x}-\beta$, the convex hull is given by

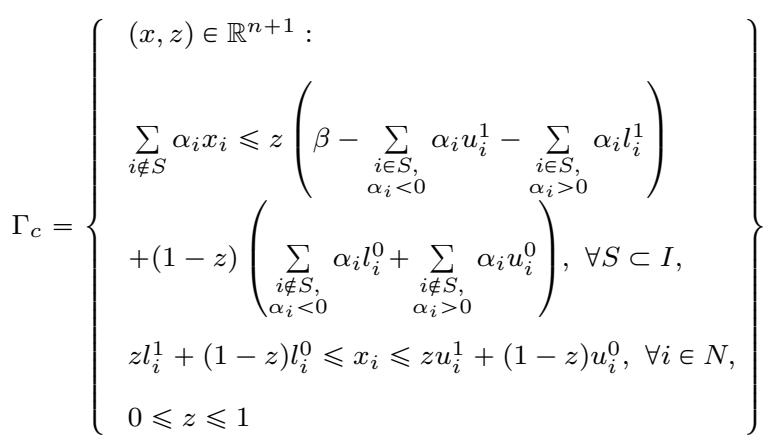

where $I=\left\{i \in\{1, \ldots, n\} \mid \alpha_{i} \neq 0\right\}$ denotes the set of variable indices appearing in the linear constraint. Observe that for $S=\varnothing$ one gets the bigM-like constraint

$$
\sum_{i \in N} \alpha_{i} x_{i} \leqslant \beta z+(1-z)\left(\sum_{\substack{i \in N \\ \alpha_{i}<0}} \alpha_{i} l_{i}^{0}+\sum_{\substack{i \in N \\ \alpha_{i}>0}} \alpha_{i} u_{i}^{0}\right)
$$

Let us emphasise that this constraint is not sufficient for defining the convex hull as shown in [21], therefore, one can strengthen the relaxation by introducing the remaining nondominated constraints. In what follows, we use the results in (7)-(10) to formulate on/off constraints in the reconfiguration framework.

\section{B. Radial Distribution Networks}

The application of the above on/off constraints principles leads to the following extension of the DistFlow relaxation (3a)-(3d) to the reconfiguration case:

$$
\begin{array}{ll}
p_{i j}=\sum_{k:(j, k) \in E} p_{j k}+\boldsymbol{r}_{i j} l_{i j}+\boldsymbol{p}_{j}^{l} & i \in N \backslash G \\
q_{i j}=\sum_{k:(j, k) \in E} q_{j k}+\boldsymbol{x}_{i j} l_{i j}+\boldsymbol{q}_{j}^{l} & i \in N \backslash G \\
v v_{j} \geqslant v v_{i}-2\left(\boldsymbol{r}_{i j} p_{i j}+\boldsymbol{x}_{i j} q_{i j}\right)+\boldsymbol{r} \boldsymbol{x}_{i j}^{2} l_{i j} & \\
-\left(\left(\boldsymbol{v}^{u}\right)^{2}-\left(\boldsymbol{v}^{l}\right)^{2}\right)\left(1-z_{i j}\right) & (i, j) \in E, j \notin G \\
v v_{j} \leqslant v v_{i}-2\left(\boldsymbol{r}_{i j} p_{i j}+\boldsymbol{x}_{i j} q_{i j}\right)+\boldsymbol{r} \boldsymbol{x}_{i j}^{2} l_{i j} & (i, j) \in E, j \notin G \\
+\left(\left(\boldsymbol{v}^{u}\right)^{2}-\left(\boldsymbol{v}^{l}\right)^{2}\right)\left(1-z_{i j}\right) & (i, j) \in E \\
p_{i j}^{2}+q_{i j}^{2} \leqslant l_{i j} v v_{i} & (i, j) \in E \\
p_{i j}^{2}+q_{i j}^{2} \leqslant l_{i j}\left(\boldsymbol{v}^{u}\right)^{2} z_{i j} & (i, j) \in E \\
p_{i j}^{2}+q_{i j}^{2} \leqslant \boldsymbol{S}_{i j}^{u} z_{i j}^{2} & i \in G \\
\sum_{(j, i) \in E} z_{j i}=0 & (i, j) \in E \\
y_{i j}=z_{i j}+z_{j i} & i \in N \backslash G \\
\sum_{(j, i) \in E} z_{j i} \leqslant 1 & i \in G \\
v v_{i}=1 & i \in N \backslash G \\
\left(\boldsymbol{v}^{l}\right)^{2} \leqslant v v_{i} \leqslant\left(\boldsymbol{v}^{u}\right)^{2} & (i, j) \in E \\
y_{i j} \in\{0,1\}, z_{i j} \in\{0,1\} &
\end{array}
$$

Equations (11a)-(11b) are identical to Equations (3a)-(3b), except for the active and reactive load $\left(\boldsymbol{p}_{j}^{l}, \boldsymbol{q}_{j}^{l}\right)$ consumed at bus $j$. Equations (111)-(11m) enforce the operational constraints on voltage at buses (6c)-(6d). Equations (11i)-(11k) enforce radial topologies. The boolean variable $z_{i j}$ indicates whether a flow is allowed from $i$ to $j$. Unlike for $y_{i j}$, there are two instances of these flow indicators per line. Equation (11i) states that incoming flows are not allowed at substations buses, Equation (11j) states that flows through an open switch are not allowed and that flows through a closed switch are unidirectional, and Equation (11k) states that the flow entering each bus comes at most from one other bus.

Equation (11h) enforces the on/off operational capacity constraint (6a). This is a direct application of the convex-hull definition (7) with $\mathbf{x}=\left(p_{i j}, q_{i j}\right), g(\mathbf{x})=x_{1}^{2}+x_{2}^{2}-\boldsymbol{S}_{i j}^{u}$ and consequently $z_{i j} g\left(\left(p_{i j}, q_{i j}\right) / z_{i j}\right)=z_{i j}\left(\frac{p_{i j}^{2}+q_{i j}^{2}}{z_{i j}^{2}}-\boldsymbol{S}_{i j}^{u}\right)$. Observe that $\mathbf{l}^{0}=\mathbf{u}^{0}=(0,0)$ hence the condition $\mathbf{l}^{0}=\mathbf{u}^{0}$ is satisfied.

Equation (11f) is identical to Equation (3d) of the DistFlow relaxation and Equation $(11 \mathrm{~g})$ is its on/off counterpart. The on/off constraint matches the quadratic form investigated in the previous subsection, whose convex hull is defined as in (8) with $\mathbf{x}=\left(p_{i j}, q_{i j}, l_{i j}\right), \boldsymbol{\alpha}=(1,1), \beta=\left(\boldsymbol{v}_{i}^{u}\right)^{2}$. Again, $\mathbf{l}^{0}=$ $\mathbf{u}^{0}=(0,0,0)$.

Finally, Equations (11c)-(11d) are the on/off counterpart of the voltage drop equation (3c) (where $\boldsymbol{r} \boldsymbol{x}_{i j}^{2}$ replaces $\boldsymbol{r}_{i j}^{2}+\boldsymbol{x}_{i j}^{2}$ ). Note that these constraints match the linear form investigated in the previous subsection. For Equation (11c) (and similarly for Equation (11d)), this leads to the bigMlike constraint in (10) with $\mathbf{x}=\left(v v_{i}, v v_{j}, p_{i j}, q_{i j}, l_{i j}\right)$, $\boldsymbol{\alpha}=\left(1,-1,-2 \boldsymbol{r}_{i j},-2 \boldsymbol{x}_{i j}, \boldsymbol{r}_{i j}^{2}+\boldsymbol{x}_{i j}^{2}\right), \beta=0, \mathbf{l}^{0}=$ $\left(\left(\boldsymbol{v}^{l}\right)^{2},\left(\boldsymbol{v}^{l}\right)^{2}, 0,0,0\right), \mathbf{u}^{0}=\left(\left(\boldsymbol{v}^{u}\right)^{2},\left(\boldsymbol{v}^{u}\right)^{2}, 0,0,0\right)$.

\section{General Distribution Networks}

We now consider the extension of the AC relaxation to reconfiguration of general (meshed) networks. Again, we make 
use of the on/off constraints principles described above. The resulting relaxation is shown below.

$$
\begin{aligned}
& p_{i j}=\boldsymbol{g}_{i j} \widetilde{v v}_{i j}^{\prime}-\boldsymbol{g}_{i j} \widetilde{w c}_{i j}-\boldsymbol{b}_{i j} \widetilde{w s}_{i j} \quad(i, j) \in E \\
& q_{i j}=-\boldsymbol{b}_{i j} \widetilde{v v}_{i j}^{\prime}+\boldsymbol{b}_{i j} \widetilde{w c}_{i j}-\boldsymbol{g}_{i j} \widetilde{w s}_{i j} \quad(i, j) \in E \\
& \widetilde{v v}_{i j}^{\prime} \geqslant \widetilde{v v}_{i}-\left(1-y_{i j}\right)\left(\boldsymbol{v}^{u}\right)^{2} \\
& \widetilde{v v}_{i j} \leqslant \widetilde{v v}_{i}+\left(1-y_{i j}\right)\left(\boldsymbol{v}^{l}\right)^{2} \\
& \left|\breve{s}_{i j}-\cos \left(\boldsymbol{\theta}^{u} / 2\right)\left(\theta_{i}-\theta_{j}\right)\right| \leqslant z_{i j}\left(\sin \left(\boldsymbol{\theta}^{u} / 2\right)\right. \\
& \left.\quad-\cos \left(\boldsymbol{\theta}^{u} / 2\right) \boldsymbol{\theta}^{u} / 2\right)+\left(1-z_{i j}\right)\left(\cos \left(\boldsymbol{\theta}^{u} / 2\right)|E| \boldsymbol{\theta}^{u}+1\right) \\
& \check{c}_{i j} \leqslant z_{i j}-\frac{1-\cos \left(\boldsymbol{\theta}^{u}\right)}{\left(\boldsymbol{\theta}^{u}\right)^{2}}\left(\left(\theta_{i}-\theta_{j}\right)^{2}+\left(1-z_{i j}\right)\left(|E| \boldsymbol{\theta}^{u}\right)^{2}\right) \\
& \check{c}_{i j} \geqslant z_{i j} \cos \left(-\boldsymbol{\theta}^{u}\right)-\left(1-z_{i j}\right) \\
& \sum_{j:(i, j) \in E} p_{i j}+\boldsymbol{p}_{i}^{l}=0 \\
& \sum_{j:(i, j) \in E} q_{i j}+\boldsymbol{q}_{i}^{l}=0 \\
& p_{i j}^{2}+q_{i j}^{2} \leqslant l_{i j}\left(\boldsymbol{v}^{u}\right)^{2} y_{i j} \\
& p_{i j}^{2}+q_{i j}^{2} \leqslant \boldsymbol{S}_{i j}^{u} y_{i j}^{2} \\
& \theta_{i}-\theta_{j} \geqslant-y_{i j} \theta^{u}-\left(1-y_{i j}\right)|E| \boldsymbol{\theta}^{u} \\
& \theta_{i}-\theta_{j} \leqslant y_{i j} \theta^{u}+\left(1-y_{i j}\right)|E| \boldsymbol{\theta}^{u} \\
& \theta_{\mathrm{ref}}=0 \\
& (5 c),(5 f)-(5 k),(6 c)-(6 d)
\end{aligned}
$$

The new power flow equations are given in (12a)-(12b). The on/off version of the squared voltage magnitude constraints are defined in (12c)-(12d). Constraints (12e), which represent the sine relaxation, are obtained by applying the convex hull formulation introduced in (9). Constraints (12f)$(12 \mathrm{~g})$ also define an on/off version of the cosine relaxation. Equations (12h)-(12i) represent Kirchhoff's law, inequalities (12j) and (12k) represent respectively the on/off version of the squared current magnitude and capacity constraints, while inequalities (12l)-(12n) encode the operational constraints on phase angles. In addition, the formulation includes constraint (5c) defining variable $\widetilde{v v}$, constraints (5f)-(5h) which encode the McCormick relaxations $\widetilde{w c}_{i j}-\widetilde{w s}_{i j}$, and constraints (5j)(5k) representing the loss equations. Finally, (6c)-(6d) states the operational constraints on voltage magnitude variables.

\section{Finding a Solution with Quality Guarantees}

The relaxations discussed above return a lower bound on the DistFlow (resp. AC) global optimal solution and compute an optimal topology in the relaxed space. This topology is then evaluated in the exact DistFlow (resp. AC) formulation by solving the non-convex model using an NLP solver. There are no on/off constraints in the latter model: instead, the set $E$ of lines is restricted to the lines $(i, j)$ such that $z_{i j}=1$ (resp. $y_{i j}=1$ ) in the optimal relaxed topology. Any feasible solution returned by the NLP solver gives us an upper bound. If such a feasible solution is returned, its objective function is compared with the lower bound to produce an optimality gap. If the gap is zero, then the lower bound is a feasible optimal solution. Otherwise, we run a heuristic MINLP solver on an exact DistFlow (resp. AC) formulation of the reconfiguration problem, and compute the optimality gap wrt. the MINLP solution.

\section{APPLICATIONS AND EXPERIMENTAL RESUlTS}

We now evaluate our approach on three reconfiguration problems: loss minimisation, load balancing, and power supply

\begin{tabular}{|c|c|c|c|c|c|}
\cline { 2 - 7 } \multicolumn{1}{c|}{} & $\begin{array}{c}\text { Obj. } \\
\text { Value }\end{array}$ & $\begin{array}{c}\text { Opt. } \\
\text { Gap }\end{array}$ & $\begin{array}{c}\text { LB. Run } \\
\text { Time }\end{array}$ & $\begin{array}{c}\text { UB. Run } \\
\text { Time }\end{array}$ & $\begin{array}{c}\text { Closed } \\
\text { Switch. }\end{array}$ \\
\hline Network & \multicolumn{5}{c|}{ Radial } \\
\hline 32-bus & 0.01395 & $0 \%$ & 2.90 & - & $32 / 37$ \\
\hline 70 -bus & 0.03016 & $0 \%$ & 7.45 & - & $68 / 76$ \\
\hline 135-bus & 0.28013 & $0 \%$ & 24.80 & - & $135 / 156$ \\
\hline 880 -bus & 0.45703 & $0 \%$ & 2886.94 & - & $873 / 900$ \\
\hline Network & \multicolumn{5}{|c|}{ Meshed } \\
\hline 32-bus & 0.01232 & $0.55 \%$ & 6.26 & 0.10 & $36 / 37$ \\
\hline 70 -bus & 0.02977 & $0.17 \%$ & 35.42 & 0.21 & $75 / 76$ \\
\hline 135-bus & 0.27079 & $3.00 \%$ & T.L. & 3.42 & $149 / 156$ \\
\hline 880 -bus & 0.45175 & $8.72 \%$ & T.L. & T.L. & $900 / 900$ \\
\hline
\end{tabular}

TABLE I. MINIMAL LOSS RESUlTS

restoration. We will provide results for the radial and meshed variants of these problems, to give a sense of the benefits that could be achieved with complex topologies.

We use the 4 networks with 32, 70, 135, and 880 buses considered by Taylor and Hover [7]. This enables us to compare our loss minimisation and load balancing results with those obtained by their QP, QCP, and SOCP approximations. We also compare with the MICP relaxation described by Jabr et. al. We reimplemented these approaches to provide fairer quantitative comparisons. For power supply restoration, we are not aware of work on convex approximations or relaxations to compare with.

All experiments were run on an AMD Opteron processor 4226 at $2.7 \mathrm{GHz}$ with 65 Gbytes of RAM and 2 Mbytes of cache. We used AMPL [22], CPLEX [8] to solve QPs and SCOPs, IPOPT [23] for NLPs, and Bonmin [24] for MINLPs. Bounds were as follows: $\boldsymbol{v}^{l}=0.9, \boldsymbol{v}^{u}=1.05$, and $\boldsymbol{\theta}^{u}=\frac{\pi}{12}$. Power supply restoration problems were run with a time limit of 30 minutes. All remaining problems were run with a time limit of $1 \mathrm{~h}$ except for the larger 880-bus instance for which it was set to $5 \mathrm{~h}$.

\section{A. Loss Minimisation}

The objective is to find the configuration minimising the total active power loss

$$
\min \sum_{(i, j) \in E} r_{i j} l_{i j}
$$

The results for the 4 networks are shown in Table I. The columns respectively report the upper bound found by NLP/MINLP, the gap (difference) with the value of the lower bound obtained with the relaxation, the run-time (sec) for the lower bound computation, the run time for the upper bound computation, and the number of switches closed in the AC solution. A Dash in the upper bound run time column indicates that the NLP found a feasible (optimal) solution and the MINLP was not run. Note that the NLP solver time is negligible. As can be seen from the table, for radial topologies, our approach finds the global optimal solution for all networks. For meshed topologies, the gap starts to become significant for the large network. The gain obtained by switching to meshed topologies ranges from at most $3 \%$ for the 135-bus network to $12 \%$ for the 32 -bus instance.

Table II compares our approach with results reported in the literature. The QP approximation of Taylor and Hover [7] gives identical results for the first 3 networks, with good run-times. However, recall that, in general, this approximation is not guaranteed to return AC-feasible solution, does not come with a provable gap with respect to the AC optimal 


\begin{tabular}{|c|c|c|c|c|c|c|c|c|c|c|}
\hline & \multicolumn{2}{|c|}{ present approach } & \multicolumn{2}{|c|}{ QP approx. [7] } & \multicolumn{2}{|c|}{ SOCP approx. [7] } & \multicolumn{2}{|c|}{ MICP relax. [14] } & \multicolumn{2}{|c|}{ Tabu search [15] } \\
\hline Network & Val & Time & Val & Time & Val & Time & Val & Time & Val & Time \\
\hline 32-bus & 0.01395 & 2.90 & 0.01395 & 0.39 & 0.01395 & 23.83 & 0.01395 & 5.23 & - & - \\
\hline 70-bus & 0.03016 & 7.45 & 0.03016 & 0.98 & 0.03016 & 769.63 & 0.03016 & 52.48 & - & - \\
\hline 135-bus & 0.28013 & 24.80 & 0.28013 & 54.74 & 0.28661 & T.L. & 0.29171 & T.L. & 0.28016 & 46.78 \\
\hline 880-bus & 0.45703 & 2886.94 & 0.45704 & T.L. & - & T.L. & mem. err. & - & - & - \\
\hline
\end{tabular}

TABLE II.

COMPARISON OF Minimal Loss Results

\begin{tabular}{|c|c|c|r|r|c|}
\cline { 2 - 7 } \multicolumn{1}{c|}{} & $\begin{array}{c}\text { Obj. } \\
\text { Value }\end{array}$ & $\begin{array}{c}\text { Opt. } \\
\text { Gap }\end{array}$ & $\begin{array}{c}\text { LB. Run } \\
\text { Time }\end{array}$ & $\begin{array}{c}\text { UB. Run } \\
\text { Time }\end{array}$ & $\begin{array}{c}\text { Closed } \\
\text { Switch. }\end{array}$ \\
\hline Network & \multicolumn{5}{c|}{ Radial } \\
\hline 32-bus & 0.04145 & $0 \%$ & 6.19 & - & $32 / 37$ \\
\hline 70-bus & 0.44126 & $0 \%$ & 35.35 & - & $68 / 76$ \\
\hline 135-bus & 0.25334 & $0 \%$ & 3600.00 & - & $135 / 156$ \\
\hline $880-$-bus & 0.10975 & $16 \%$ & T.L. & - & $873 / 900$ \\
\hline Network & \multicolumn{5}{|c|}{ Meshed } \\
\hline 32-bus & 0.03511 & $0.59 \%$ & 16.25 & 52.76 & $34 / 37$ \\
\hline 70-bus & 0.41840 & $0.30 \%$ & 874.17 & 484.90 & $72 / 76$ \\
\hline 135-bus & 0.21494 & $0.76 \%$ & T.L. & T.L. & $148 / 156$ \\
\hline 880-bus & 0.10975 & $15.40 \%$ & T.L. & T.L. & $873 / 900$ \\
\hline
\end{tabular}

TABLE III. LOAD BALANCING RESULTS

solution, and does not apply to other reconfiguration problems such as load balancing. Moreover, for the larger network, our approach finds the optimal solution in a fraction of the time taken by the QP approximation. The table also shows that the SOCP approximation [7], which is more powerful than the QP approximation, suffers from computational inefficiencies. Unlike these approximations, the MICP relaxation by Jabr et al. [14] provides optimality guarantees. However, this formulation lacks an efficient modelling of the on/off constraints which is reflected in its weaker computational performance. Note that it fails to converge to the optimal solution on the 135-bus instance and raises a memory error on the 880-bus benchmark. The table also mentions results obtained with Tabu search [15] which returns AC feasible solutions and achieves a reasonable compromise between quality and efficiency. The lower bound provided by our approach enables us to assess the optimality of meta-heuristics and approximation methods, showing, e.g., that the optimality gap of the QP approximation is 0 for the 3 first networks.

\section{B. Load Balancing}

As described in [7], the objective is to find the configuration minimising the maximum line capacity usage ratio, that is, minimising the ratio $t$ defined by the additional constraint

$$
p_{i j}^{2}+q_{i j}^{2} \leqslant \boldsymbol{S}_{i j}^{u} t
$$

Table III shows the results for our approach. The first observation is that the new objective function is more difficult to optimise than loss minimisation. The second is that we nevertheless manage to find zero gap solutions for medium size radial networks and good solutions (with a gap less than $1 \%$ ) for the three first meshed networks. The third observation is that the benefits of meshed topologies are more substantial in this case, with $15 \%$ improvement for both the 32-bus and 135-bus networks, and 5\% for the 70-bus.

Table IV compares our results on the load balancing problem with those obtained with the QCP and SOCP approximations in [7]. Note that the former returns an infeasible topology on the 70-bus instance while the latter computes suboptimal solutions on all benchmarks. On the 880-bus instance, the QCP approximation returns the best solution, with an optimality gap of $8 \%$, as can be established using our lower bound.

\begin{tabular}{|c|r|r||r|r||r|r|}
\cline { 2 - 7 } \multicolumn{1}{c|}{} & \multicolumn{2}{c||}{ present approach } & \multicolumn{2}{c||}{ QCP approx. [7] } & \multicolumn{2}{c|}{ SOCP approx. [7] } \\
\hline Network & Val & Time & \multicolumn{1}{c|}{ Val } & \multicolumn{1}{c|}{ Time } & Val & \multicolumn{1}{c|}{ Time } \\
\hline 32-bus & 0.04145 & 6.19 & 0.04146 & 4.42 & 0.04146 & 9.73 \\
\hline 70-bus & 0.44126 & 35.35 & inf. & 778.31 & 0.44879 & 1690.25 \\
\hline 135-bus & 0.25334 & T.L. & 0.25334 & T.L. & 0.28380 & T.L. \\
\hline 880-bus & 0.10975 & T.L. & 0.10116 & T.L. & 0.14238 & T.L. \\
\hline
\end{tabular}

TABLE IV. COMPARISON OF LOAD BALANCING RESULTS

\section{Power Supply Restoration}

Power supply restoration consists in reconfiguring the network to isolate faults and resupply as much of the existing load as possible. Let $F \subseteq N$ denotes the set of faulty buses. To simplify notations, we assume that faults only occur on buses, however the model can be simply extended to handle line faults. For each bus $i \in N$, we introduce a new boolean variable $f_{i}$ which is 1 iff $i$ is fed, indicating that there exists a path consisting of closed switches from a substation bus to $i$. If bus $i \in N \backslash G$ is fed, then all its load $\left(\boldsymbol{p}_{i}^{l}, \boldsymbol{q}_{i}^{l}\right)$ must be supplied. In supply restoration problems, it is not possible to feed all buses: faulty buses must not be fed, and sometimes other buses cannot be fed either as a result of the interplay between faults and operational constraints (in particular line capacity).

Handling power supply restoration requires a slight change in Equations (11a)-(11b) and (12h)-(12i), we replace occurrences of the loads $\boldsymbol{p}_{i}^{l}$ and $\boldsymbol{q}_{i}^{l}$ with $f_{i} \boldsymbol{p}_{i}^{l}$ and $f_{i} \boldsymbol{q}_{i}^{l}$, respectively, to account for the fact that not all buses are fed. For instance, equation (11a) becomes

$$
p_{i j}=\sum_{k:(j, k) \in E} p_{j k}+\boldsymbol{r}_{i j} l_{i j}+f_{j} \boldsymbol{p}_{j}^{l} \quad i \in N \backslash G
$$

The only other change is the addition of the following two operational constraints,

$$
\begin{aligned}
& f_{i}=0 \quad i \in F \\
& y_{i j} \leqslant f_{i}-f_{j}+1 \quad(i, j) \in E
\end{aligned}
$$

Constraint (14a) enforces that no faulty bus is fed. Constraint (14b) states that if a line switch is closed, the connected buses must be either both fed or both unfed; this forces the separation of the fed from the unfed regions via open switches.

A lexicographic objective function is defined by first maximising the load supplied and then minimising the number of switching operations, that is,

$$
\max M\left(\sum_{i \in N} f_{i} \boldsymbol{p}_{i}^{l}\right)-\sum_{(i, j) \in E}\left|y_{i j}-\boldsymbol{y}_{i j}^{0}\right|
$$

with $M>|E| / \min \left(p_{i}^{l}, 1\right), \forall i \in N$, and where $\boldsymbol{y}_{i j}^{0}$ represents the position of switch $(i, j) \in E$ prior to reconfiguration.

Table $\mathrm{V}$ shows the results obtained using our approach for each of the 4 networks and an increasing number $n$ of faults between 1 and 3. For radial topologies, each row of the table averages the results over $\min \left(|N|^{n}, 200\right)$ random fault scenarios. The same applies for meshed topologies, except for 


\begin{tabular}{|c|r|r|r||r|r|r|}
\cline { 2 - 7 } \multicolumn{1}{c|}{} & \multicolumn{7}{c||}{ Radial } & \multicolumn{3}{c|}{ Meshed } \\
\cline { 2 - 7 } \multicolumn{1}{c|}{} & Load & Time & \multicolumn{1}{c|}{ Ops. } & \multicolumn{1}{c|}{ Load } & Time & Ops. \\
\hline Network & \multicolumn{7}{|c|}{ 1 Fault } \\
\hline 32-bus & $89.53 \%$ & 0.70 & 2.79 & $90.42 \%$ & 1.29 & 2.33 \\
\hline 70 -bus & $91.75 \%$ & 11.72 & 2.91 & $95.89 \%$ & 335.04 & 2.88 \\
\hline 135-bus & $98.97 \%$ & 3.55 & 2.65 & $98.97 \%$ & 23.51 & 2.18 \\
\hline 880-bus & $99.62 \%$ & 40.94 & 2.5 & $96.77 \%$ & 1237.14 & 3.68 \\
\hline Network & \multicolumn{7}{|c|}{ 2 Faults } \\
\hline 32-bus & $75.82 \%$ & 1.04 & 5.07 & $77.59 \%$ & 2.19 & 4.5 \\
\hline 70 -bus & $87.12 \%$ & 22.54 & 5.23 & $91.50 \%$ & 455.18 & 5.68 \\
\hline 135-bus & $97.90 \%$ & 4.26 & 5.09 & $97.90 \%$ & 21.18 & 4.19 \\
\hline 880-bus & $99.05 \%$ & 73.60 & 4.80 & $97.37 \%$ & 1446.63 & 6.14 \\
\hline Network & \multicolumn{7}{|c|}{3 Faults } \\
\hline 32-bus & $66.73 \%$ & 0.88 & 6.86 & $68.83 \%$ & 1.91 & 6.53 \\
\hline 70 -bus & $79.64 \%$ & 31.03 & 7.45 & $85.03 \%$ & 365.55 & 8.50 \\
\hline 135-bus & $96.98 \%$ & 24.74 & 7.70 & $96.89 \%$ & 22.59 & 6.28 \\
\hline 880-bus & $98.88 \%$ & 58.17 & 7.31 & $99.01 \%$ & 1225.08 & 7.12 \\
\hline
\end{tabular}

TABLE V. POWER SUPPLY RESTORATION RESULTS

\begin{tabular}{|c|c|c|c||r|r|c|}
\hline Nb. Faults: & 1 & 2 & 3 & 1 & 2 & 3 \\
\hline Network & \multicolumn{3}{|c|}{ Radial } & \multicolumn{3}{|c|}{ Meshed } \\
\hline 32-bus & $15 \%$ & $20 \%$ & $30 \%$ & $6 \%$ & $14 \%$ & $22 \%$ \\
\hline 70-bus & $55 \%$ & $75 \%$ & $81 \%$ & $21 \%$ & $43 \%$ & $62 \%$ \\
\hline 135-bus & $9 \%$ & $16 \%$ & $24 \%$ & $0 \%$ & $0.5 \%$ & $0.5 \%$ \\
\hline 880-bus & $3 \%$ & $7 \%$ & $9 \%$ & $2 \%$ & $4 \%$ & $4 \%$ \\
\hline
\end{tabular}

TABLE VI. PRoBlems With INFEASIBLE DC SOLUTIONS

the 880-bus instance where, given the increased computational difficulty, only 50 scenarios were used. This results in a total of 3624 problems being solved. The columns show the average fraction of active load served after the restoration, the average run-time, and the average number of switching operations involved. The average run-time accounts for both the relaxation and the NLP solving time. For radial networks, the relaxation provided an optimal feasible solution in over $99 \%$ of cases and a near-optimal solution on the remaining scenarios, within a minute of computation time on average.

In the meshed case, there is a computational cost associated with the AC power flow model, and global optimality guarantees are only obtained on $80 \%$ of instances. Observe that, on average, the percentage of served load increases in comparison to the radial networks.

Finally, Table VI highlights the weakness of the widely used linear DC model as a substitute for convex relaxations, reporting the percentage of instances for which the DC optimal solution could not be converted into an AC feasible one.

\section{Conclusion}

This paper presents tractable convex relaxations of reconfiguration problems for radial and meshed topologies. In combination with generic MINLP solvers they lead to provably optimal or near-optimal, AC feasible solutions for a wide range of reconfiguration problems. The benefits of a right representation of the on/off constraints are highlighted in the numerical experiments section where the present approach competes with approximate methods while offering provable optimality bounds. An important and challenging aspect of future work is to account for transient phenomena which occur after switching operations in the presence of distributed generation, and which may compromise the stability of the network.

\section{ACKNOWLEDGMENT}

We would like to thank Carleton Coffrin, Alban Grastien and Pascal Van Hentenryck for their useful comments.
NICTA is funded by the Australian Government through the Department of Communications and the Australian Research Council through the ICT Centre of Excellence Program.

\section{REFERENCES}

[1] M. E. Baran and F. F. Wu, "Network reconfiguration in distribution systems for loss reduction and load balancing," IEEE Trans. Power Delivery, vol. 4, no. 2, 1989.

[2] H.-D. Chiang and R. Jean-Jumeau, "Optimal network reconfigurations in distribution systems. i. a new formulation and a solution methodology," IEEE Trans. Power Delivery, vol. 5, no. 4, 1990.

[3] S. Toune, H. Fudo, T. Genji, Y. Fukuyama, and Y. Nakanishi, "Comparative study of modern heuristic algorithms to service restoration in distribution systems," IEEE Trans. Power Syst., vol. 17, no. 1, 2002.

[4] U. G. Knight, Power systems engineering and mathematics, by U. G. Knight. Pergamon Press Oxford, New York, 1972.

[5] B. Stott, J. Jardim, and O. Alsac, "Dc power flow revisited," IEEE Trans. Power Syst., vol. 24, no. 3, 2009.

[6] C. Coffrin and P. Van Hentenryck, "A Linear-Programming Approximation of AC Power Flows," INFORMS J. Computing, To appear.

[7] J. Taylor and F. Hover, "Convex models of distribution system reconfiguration," IEEE Trans. Power Syst., vol. 27, no. 3, 2012.

[8] IBM, "ILOG CPLEX software," http://www.ibm.com/, 2012.

[9] C. Coffrin, P. Van Hentenryck, and R. Bent, "Smart Load and Generation Scheduling for Power System Restoration," Proc. IEEE Power \& Energy Society General Meetings (PES), 2012.

[10] J. Taylor and F. Hover, "Linear relaxations for transmission system planning," IEEE Trans. Power Syst., vol. 26, no. 4, 2011.

[11] M. Farivar, C. Clarke, S. Low, and K. Chandy, "Inverter var control for distribution systems with renewables," in Proc. 2nd IEEE Int. Conf. on Smart Grid Communications (SmartGridComm), 2011.

[12] J. Lavaei and S. Low, "Zero duality gap in optimal power flow problem," IEEE Trans. Power Syst., vol. 27, no. 1, 2012.

[13] C. Hijazi, C. Coffrin, and P. Van Hentenryck, "Convex quadratic relaxations of nonlinear programs in power systems," Optimization OnLine, aug. 2013.

[14] R. Jabr, R. Singh, and B. Pal, "Minimum loss network reconfiguration using mixed-integer convex programming," IEEE Trans. nPower Syst., vol. 27, no. 2, 2012.

[15] M. Guimarães and C. Castro, "Reconfiguration of distribution systems for loss reduction using tabu search," in Proc. Power Systems Computation Conference (PSCC), 2005.

[16] S. Sojoudi and J. Lavaei, "Network Topologies Guaranteeing Zero Duality Gap for Optimal Power Flow Problem," Proceedings of the 2012 IEEE Power \& Energy Society General Meetings (PES), 2012.

[17] M. Farivar and S. Low, "Branch flow models: Relaxations and convexification," abridged version in IEEE CDC, Dec 2012.

[18] L. Liberti, "Reduction constraints for the global optimization of nlps," Int. Trans. Operational Research, vol. 11, no. 1, 2004.

[19] I. Grossmann and S. Lee, "Generalized convex disjunctive programming: Nonlinear convex hull relaxation," Computational Optimization and Applications, vol. 26, no. 1, 2003.

[20] O. Günlük and J. Linderoth, "Perspective reformulation and applications," in Mixed Integer Nonlinear Programming, ser. The IMA Volumes in Mathematics and its Applications. Springer, 2012, vol. 154.

[21] H. Hijazi, P. Bonami, G. Cornujols, and A. Ouorou, "Mixed-integer nonlinear programs featuring on/off constraints," Computational Optimization and Applications, vol. 52, no. 2, 2012.

[22] R. Fourer, D. M. Gay, and B. W. Kernighan, AMPL: A Modeling Language for Mathematical Programming. Duxbury Press, 2002.

[23] A. Wächter and L. T. Biegler, "On the implementation of a primaldual interior point filter line search algorithm for large-scale nonlinear programming," Mathematical Programming, vol. 106, no. 1, 2006.

[24] P. Bonami, L. T. Biegler, A. R. Conn, G. Cornuejols, I. E. Grossmann, C. D. Laird, J. Lee, A. Lodi, F. Margot, N. Sawaya, and A. Wächter, "An algorithmic framework for convex mixed integer nonlinear programs," Discrete Optimization, vol. 5, no. 2, 2008. 